\title{
A novel approach to treatment of haemorrhagic cerebral vascular accidents
}

\author{
Godeanu S.C.' , Stanciulescu E.-L.', Popescu M.' ${ }^{2}$, Grintescu I.M.' \\ 'Emergency Clinical Hospital of Bucharest, Dept of Anaesthesiology \& Intensive Care, \\ Bucharest, Romania, ${ }^{2}$ Clinical Hospital Colentina, Dermatology, Bucharest, Romania
}

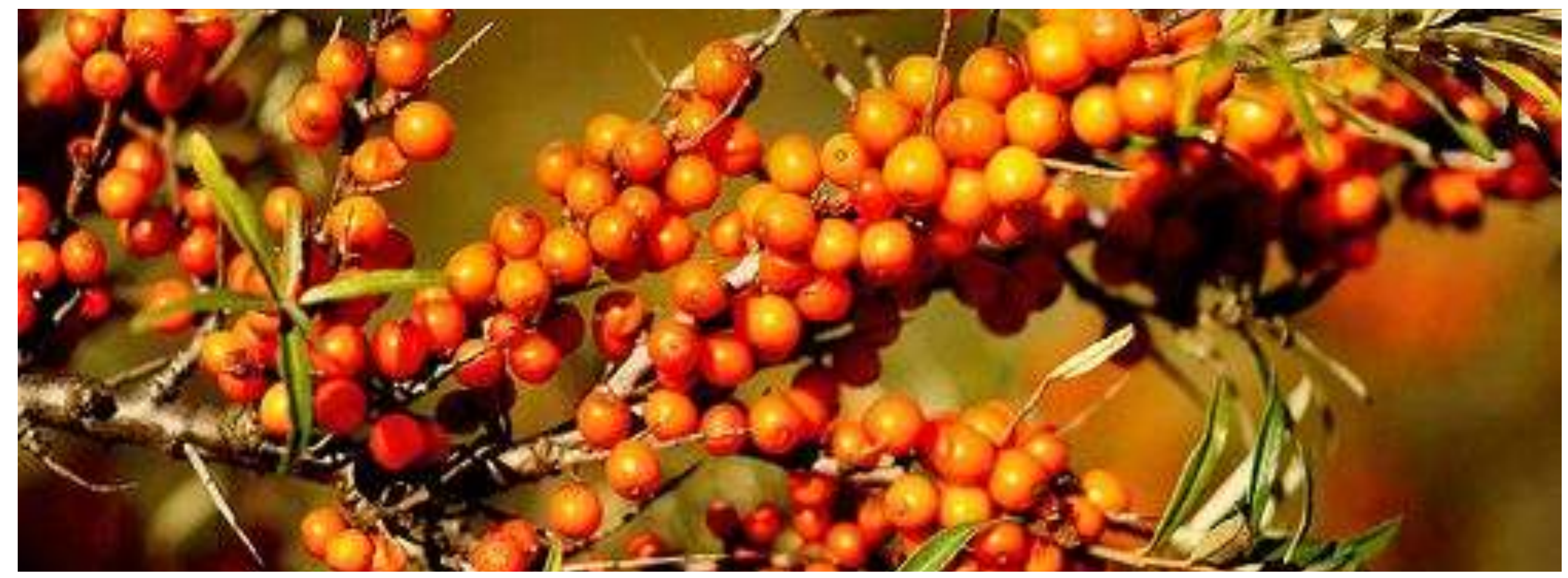

Cerebral vascular accidents (CVA) represent one of the most common causes of death in Europe, and it is also a major cause of severe disability and morbidity. The main risk factor is high blood pressure, studies have shown that oxidative stress is significantly higher in this group of patients. The research and introduction of novel therapies associated with the current allopathic medicine treatments can improve the patients outcome.

A 64-year old female patient who was a well known, poor-controlled hypertensive patient was admitted to our clinic in aphasia, very drowsy, GCS = 7 (EIVIM5), divergent strabismus of both eyes, bilateral eyelid ptosis, $\mathrm{BP}=220 / 120 \mathrm{mmHg}$. An MRI scan was performed which has shown a haemorrhagic area in the central area of the mesencephalon, extending into the cerebral peduncles and into both thalamic regions, measuring $1.5 / 3 \mathrm{~cm}$, and also numerous cavernomas in both bazal nuclei, highly suggestive of a ruptured cavernoma.

Standard allopathic treatment as per protocol for haemorrhagic CVA was commenced, along with a phytotherapy treatment, comprised of $10 \mathrm{ml} / \mathrm{kg}$ Hippophae rhamnoides fresh juice three times daily, and six tablets of a Urtica Dioica $200 \mathrm{mg}$, Taraxacum Officinalis $80 \mathrm{mg}$, Allium Ursinum $30 \mathrm{mg}$, Ranunculus Ficaria $30 \mathrm{mg}$, Rumex Alpinus $30 \mathrm{mg}$, two tablets three times daily, all being administered on the nasogastric tube. Legal consent has been obtained from the legal caregiver.
The patient's neurological status improved gradually, being taken off the mechanical ventilation a week after admission. A CT scan was performed at 14 days following admission which showed massive resorbtion of the mesencephalic haemorrhage. The patient was discharged at fourty-five days since admission, alert, with no neurological deficit apart from the eyelid ptosis.

Hippophae rhamnoides contains over 190 bioctive substances, (flavonoids, quercetin, isorhamnetin, carotenoids) and has a very strong antioxidant effect, and also an antithrombotic, neuroprotective, immunostimulating effect. It has been shown to increase capillary resistance and has some antibacterial effects as well.

We believe that the phytotherapeutic cocktail has contributed to the improvement of the cerebral circulation, stabilisation of the vascular endotelium, decrease of the cerebral oedema and has demonstrated a powerful antiinflammatory and antioxidant effect.The nervous system is very sensitive to oxidative stress (evaluated through ceruloplasmin and malondialdehide serum levels) and has very scanty defense systems (glutation peroxidase in very low levels). Following the complementary treatment using the phytotherapeutic cocktail we have noticed an obvious clinical improvement due to its strong antioxidant and neuroprotective effects. Further research is however needed to quantify these benefits. No side effects to the phytotherapeutic medication was observed. 